\title{
Therapeutic effect of intravitreal injections of ranibizumab for the treatment of macular choroidal neovascularization caused by pathological myopia
}

\author{
LEIBING JI $^{1}$, WENJUAN LV ${ }^{1}$, YUN XIAO ${ }^{1}$, ZHENGHUA XU ${ }^{1}$, XIAOLING ZHANG ${ }^{1}$ and WEI ZHANG ${ }^{2}$ \\ ${ }^{1}$ PLA Ophthalmic Center, No. 474 Hospital of the Chinese PLA, Urumqi, Xinjiang Uyghur Autonomous Region 830011; \\ ${ }^{2}$ The Graduate School, Shihezi University, Shihezi, Xinjiang 832000, P.R. China
}

Received September 3, 2014; Accepted April 17, 2015

DOI: 10.3892/etm.2015.2625

\begin{abstract}
The aim of the present study was to evaluate the clinical efficacy and safety of intravitreal ranibizumab injections for the treatment of macular choroidal neovascularization (CNV) caused by pathological myopia. Between one and four intravitreal injections of ranibizumab were administered to 61 eyes from 61 patients who were diagnosed with macular CNV caused by pathological myopia. Following injection, the best-corrected visual acuity (BCVA), central macular thickness (CMT) and fundus fluorescein angiography (FFA) findings were evaluated monthly for a period of 6 months. Among the 61 eyes, 10 eyes received one injection, 44 received two injections, six received three injections and one received four injections (average, 1.97 injections). The BCVA was $0.02 \pm 0.01$ prior to treatment and $0.30 \pm 0.03$ subsequent to treatment, and this difference was statistically significant $(\mathrm{P}<0.01)$. The CMT was reduced by an average of $45.1 \mu \mathrm{m}$. Regarding the FFA results, 56 eyes had no CNV fluorescence leakage and five eyes had CNV fluorescence leakage following treatment; however, the intensity of CNV fluorescence leakage in the five eyes following treatment was lower than that prior to treatment. As a treatment for pathological myopia-induced macular CNV, intravitreal injections of ranibizumab may improve eyesight as well as the macular retinal tissue structure; thus, this is a safe and effective treatment method.
\end{abstract}

\section{Introduction}

With the continuous development of human society and increases in the human life span, myopia has become a

Correspondence to: Professor Yun Xiao, PLA Ophthalmic Center, No. 474 Hospital of the Chinese PLA, 754 Beijing Road, Urumqi, Xinjiang Uyghur Autonomous Region 830011, P.R. China

E-mail: yunxiaocn@126.com

Key words: choroidal neovascularization, pathological myopia, ranibizumab severe vision-threatening disease. Furthermore, choroidal neovascularization (CNV), which is caused by pathological myopia, has become a common complication resulting in blindness (1). Pathological myopia has exhibited a trend toward increased incidence in Asian populations, and it is particularly common in China and Japan $(2,3)$; therefore, research toward an effective treatment of pathological myopia-induced CNV would have a profound effect on improving survival quality. Pathological myopia-induced macular CNV was previously treated by photodynamic therapy (PDT); however, the majority of studies showed that the reformation of macular CNV following PDT led to vision reduction in over one-third of patients or, even if vision was stable following the treatment, it was not improved $(4,5)$. The widespread use of anti-vascular endothelial growth factor (VEGF) drugs in the field of age-related macular degeneration (AMD) has provided a novel approach for the treatment of CNV (6). Although the drug bevacizumab has not yet obtained clinical recognition, it has received some distinction in the treatment of pathological myopia-induced CNV (7). By contrast, ranibizumab (Lucentis ${ }^{\circledR}$; Novartis Pharma AG, Basel, Switzerland), which has been clinically registered for the treatment of AMD, has been described as a treatment for pathological myopia-induced CNV in only a few reports (8-10).

The aim of the present study was to evaluate the safety and efficacy of intravitreal injections of ranibizumab in the treatment of CNV inside the macula lutea of patients with pathological myopia. The present research was based on the different mechanisms of ranibizumab in the treatment of macular and non-macular CNV caused by pathological myopia. The former condition was evaluated in this study, due to the fact that this condition is normally associated with smaller lesions than those of AMD CNV, and the incidence rate of macular CNV caused by pathological myopia is higher than that of non-macular CNV $(11,12)$. For the present study, the random prospective method without controls was adopted. A total of 61 eyes from 61 patients diagnosed with macular pathological myopia-induced CNV were selected to receive $0.05 \mathrm{ml}$ intravitreal injection(s) of ranibizumab. The monitored follow-up period was 6 months, and the curative effects were evaluated. 


\section{Materials and methods}

General information. A total of 61 eyes from 61 patients, who were attending the No. 474 Hospital of the Chinese PLA (Urumqi, China) between August 2012 and January 2013, were included in this study. The patients had been diagnosed with pathological myopia-induced macular CNV following fundus fluorescein angiography (FFA) and optical coherence tomography (OCT) examination. The patients included 22 men (22 eyes) and 39 women (39 eyes) aged 20.2-49.6 years, with a mean age of 25.2 years. The diopter values ranged from -6.50 to $-16.00 \mathrm{D}$ (average, $10.50 \pm 4 \mathrm{D}$ ), and the average eye axis was $27.9 \mathrm{~mm}$.

Patients with systemic and ocular surgery contraindications were excluded. Intravitreal injections of $0.05 \mathrm{ml}$ ranibizumab (Novartis Pharma AG) were administered under local anesthesia to 61 eyes from 61 patients in the hospital; the number of injections administered to each patient varied between one and four. This study was conducted in accordance with the Declaration of Helsinki and with approval from the Ethics Committee of the No. 474 Hospital of the Chinese PLA. Written informed consent was obtained from all participants.

Intravitreal injection. Prior to the intravitreal injection, the patients were instructed to use tobramycin and dexamethasone ophthalmic suspension (TobraDex ${ }^{\circledR}$; Alcon-Couvreur N.V., Puurs, Belgium) and levofloxacin eye drops (Shandong Bausch and Lomb Freda Pharmaceutical Co. Ltd., Jinan, China) four times per day for 3 days. On the day of the procedure, the patient entered the sterile laminar flow procedure room and their personal information was checked. Each patient received three local anesthesia treatments with $0.4 \%$ oxybuprocaine hydrochloride eye drops (Towering Pharmaceutical Co., Ltd., Jiangsu, China) $10 \mathrm{~min}$ before the procedure. Following the disinfection and washing of the eye with diluted gentamicin liquid (Henan Furen Pharmaceutical Co., Ltd., Zhenghou, China), a 1-ml TB needle (Jiangsu Zhengkang Medical Equipment Co., Ltd., Changzhou, China) was vertically punctured into the vitreous cavity from $4.0 \mathrm{~mm}$ lateral to the lower limbus. When it was confirmed that the TB needle had entered the vitreous cavity and had no contact with the lens posterior capsule, $0.05 \mathrm{ml}$ ranibizumab was slowly injected. Following the withdrawal of the needle, the needlepoint was gently pressed with a wet cotton swab for 1-2 min, and the eye was subsequently coated with TobraDex oculentum and sterile gauze. The day after the surgery, the patients were evaluated for the presence of subconjunctival hemorrhage, anterior chamber reaction or endophthalmitis, increased intraocular pressure, complicated cataract, retinal hole, retinal detachment and other complications. If the patient was found to have a complication, it was treated in a timely manner. Following the procedure, TobraDex and levofloxacin eye drops were used in the treated eye for 3 consecutive days, and the patients were followed-up for 6 months thereafter.

Standards for repeated injections. The following standards were used to determine the requirement for repeated injections: i) The visual acuity decreased by 0.05 or conscious vision decline occurred; ii) a new hemorrhage was observed in the macular area; iii) FFA revealed that the leakage area had increased or a new leakage was found; iv) OCT revealed an incomplete fibrotic or predominantly fibrotic $\mathrm{CNV}$; and v) effusion from under or inside the retinal hole was unchanged or increased. The patient received a repeat injection if any of these situations occurred.

Observation items. The main observation items were as follows: i) The number of intravitreal ranibizumab injections; ii) the average best-corrected visual acuity (BCVA); iii) the macular retinal thickness (CMT) as observed by OCT; and iv) evaluation of the presence of apparent macular persistent $\mathrm{CNV}$ fluorescent leakage, as observed using FFA. In addition, the following secondary items were observed: i) The presence of subconjunctival hemorrhage; ii) the occurrence of an infectious anterior chamber reaction or endophthalmitis; iii) an increase in intraocular pressure; iv) the presence of a cataract complication; and v) retinal breaks or detachment.

Statistical analysis. Using SPSS software (version 15.0; SPSS Inc., Chicago, IL, USA), the mean BCVA and the CMT shown by OCT were compared using the paired-samples t-test or multivariate analysis of variance. Data are presented as the mean \pm standard deviation. $\mathrm{P}<0.05$ was considered to indicate a statistically significant difference.

\section{Results}

Main observation items. The numbers of intravitreal injections of ranibizumab were as follows: One injection in 10 eyes, two injections in 44 eyes, three injections in six eyes and four injections in one eye (average, 1.97 injections). The average BCVA was $0.02 \pm 0.01$ prior to treatment and $0.30 \pm 0.03$ subsequent to treatment, and $88.5 \%$ of cases (54/61) showed an improved and stable visual acuity. OCT revealed that the CMT was reduced by an average of $45.1 \mu \mathrm{m}$ among the patients. Using FFA to display macular fluorescein leakage, 56 cases were found to have no evident CNV fluorescence leakage following treatment, whereas five cases with CNV had fluorescence leakage following treatment; however, the leakage intensity subsequent to treatment was lower compared with that prior to treatment in these cases (Table I and Fig. 1).

Secondary observation items. Analysis of the secondary observation items revealed that no eyes had subconjunctival hemorrhage; one eye had an anterior chamber reaction with no endophthalmitis; three eyes had increased intraocular pressure (average, $5 \mathrm{mmHg}$ ), including one case in which the intraocular pressure increased up to $23.2 \mathrm{mmHg}$; no cases had a cataract complication; and no cases had retinal breaks or detachments.

\section{Representative cases}

Case 1. A 40-year-old female patient was admitted to the hospital in October 2012 with the main complaint that 'the vision of the left eye had been decreased for 2 months'. During the physical examination, the vision of the left eye was checked: Naked eye, $0.02 /$ correction with the original glasses $(-8.00 \mathrm{D}) / 0.4$ and ocular anterior segment (-). Vitreous opacities were observed. While the optic disc of the eyeground had a clear boundary and light color, myopic atrophy was evident. The retina exhibited a leopard pattern, and thick bleeding 
Table I. Numerical comparison of pathological myopic macular choroidal neovascularization before and after treatment.

\begin{tabular}{lccc}
\hline Parameter & Before treatment & After the final treatment & P-value \\
\hline Average best-corrected visual acuity $(\mu \mathrm{m})^{\mathrm{a}}$ & $0.02 \pm 0.01$ & $0.30 \pm 0.03$ & $<0.01$ \\
Average macular retinal thickness $(\mu \mathrm{m})^{\mathrm{a}}$ & $395.9 \pm 143.9$ & $240.8 \pm 71.7$ & 5
\end{tabular}

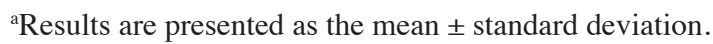

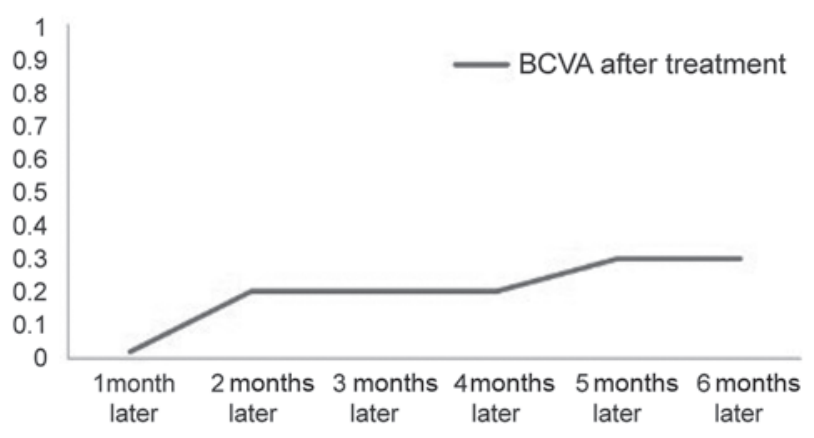

Figure 1. Average BCVA curve following the treatment of pathological myopic macular choroidal neovascularization. BCVA, best-corrected visual acuity.

lesions were apparent under the central fovea of the macula. The FFA and OCT findings of the fundus oculi are shown in Fig. 2A-C. The left eye was administered a 0.05-ml intravitreal injection of ranibizumab, and a physical examination was performed 1 month later. At this examination, the eyesight in the left eye was as follows: Naked eye, $0.04 /$ correction with the original glasses (-8.00 D)/0.5 and ocular anterior segment (-). Vitreous opacities were observed. Although the optic disc of the eyeground had a clear boundary and light color, myopic atrophy was apparent. The leopard pattern of the retina remained but the range of the thick bleeding lesions under the central fovea of the macula was reduced. The FFA and OCT findings of the fundus oculi are shown in Fig. 2D and E. The vision of the left eye was rechecked 3 and 6 months later: Naked eye, $0.04 /$ correction with the original glasses $(-8.00 \mathrm{D}) / 0.6$ and ocular anterior segment (-). Vitreous opacities were apparent. The eyeground FFA indicated that the neovascular leakage of the macula lutea had disappeared, as the high fluorescence leakage observed previously had become a weak fluorescence leakage. OCT revealed that the macular CNV was basically fibrotic, and the reflective optical bands had changed from low and middle signals to high signals (Fig. 2F-I).

Case 2. A 27-year-old male patient was admitted to the hospital in November 2012 with the main complaint that 'the substances seen by the right eye had been deformed for 1 month'. The physical examination upon admission revealed that the vision of the left eye was as follows: Naked eye, index $(10 \mathrm{~cm}) /$ correction with the original glasses (-12.00 D)/0.02 and ocular anterior segment (-). The crystal cortex exhibited slight turbidity, and vitreous opacities were apparent. The optic disc of the eyeground had a clear boundary and light color, but myopic atrophy was evident. The retina exhibited a leopard pattern, with apparent chorioretinal atrophy in the macula lutea. The eyeground FFA and OCT findings are shown in Fig. 3A-D. The left eye was administered a $0.05-\mathrm{ml}$ intravitreal injection of ranibizumab, and a physical examination was performed 1 month later. This examination revealed the following in the left eye: Naked eye, index $(10 \mathrm{~cm}) /$ correction with the original glasses $(-12.00 \mathrm{D}) / 0.08$ and ocular anterior segment (-). The crystal cortex exhibited slight turbidity, and vitreous opacities were apparent. While the optic disc of the eyeground had a clear boundary and light color, myopic atrophy was evident; additionally, the retina exhibited a leopard pattern, and chorioretinal atrophy was apparent in the macula lutea. No significant differences were detected in best-corrected visual, FFA and OCT during subsequent 5-month follow-up period (Fig. 3E-H).

The aforementioned two cases were treated with an intravitreal injection of ranibizumab. Subsequently, FFA and OCT revealed that the active lesions had become inactive, vision was increased, the degree of fibrosis of the CNV was strengthened or the CNV was completely fibrotic and the retinal thickness of the macula lutea was decreased.

\section{Discussion}

Anti-VEGF therapy has become an important treatment for $\mathrm{CNV}$, and evidence for its usefulness has been increasing (13). At present, six main types of medical treatment are used for pathological myopia-induced macular CNV: Retinal laser photocoagulation, surgical resection, macular translocation (14), PDT, anti-VEGF drugs and Traditional Chinese Medicine. Compared with the other treatments, anti-VEGF drugs have significant advantages. Retinal laser photocoagulation and surgical treatment are likely to cause irreversible structural damage to the macular tissues. Ladas et al (15) stated that PDT is not ideal for improving eyesight and that it may induce choroidal capillary non-perfusion. In addition, certain individual cases may exhibit direct or indirect lacquer crack-like lesions following PDT treatment, and these lesions can easily cause CNV (15). Although improvements have been reported following treatment with Traditional Chinese Medicine, large-sample observations and reproducibility are lacking in these studies. The anti-VEGF drugs currently on the market include pegaptanib, bevacizumab, triamcinolone and ranibizumab, but ranibizumab (Lucentis) has significant advantages compared with the other drugs, and it is the only anti-VEGF drug that has been approved for intraocular injection. Lucentis, which is a VEGF inhibitor co-developed by Genentech and Novartis, was designed as a Fab fragment, and this fragment can penetrate the retinal layer more easily than 

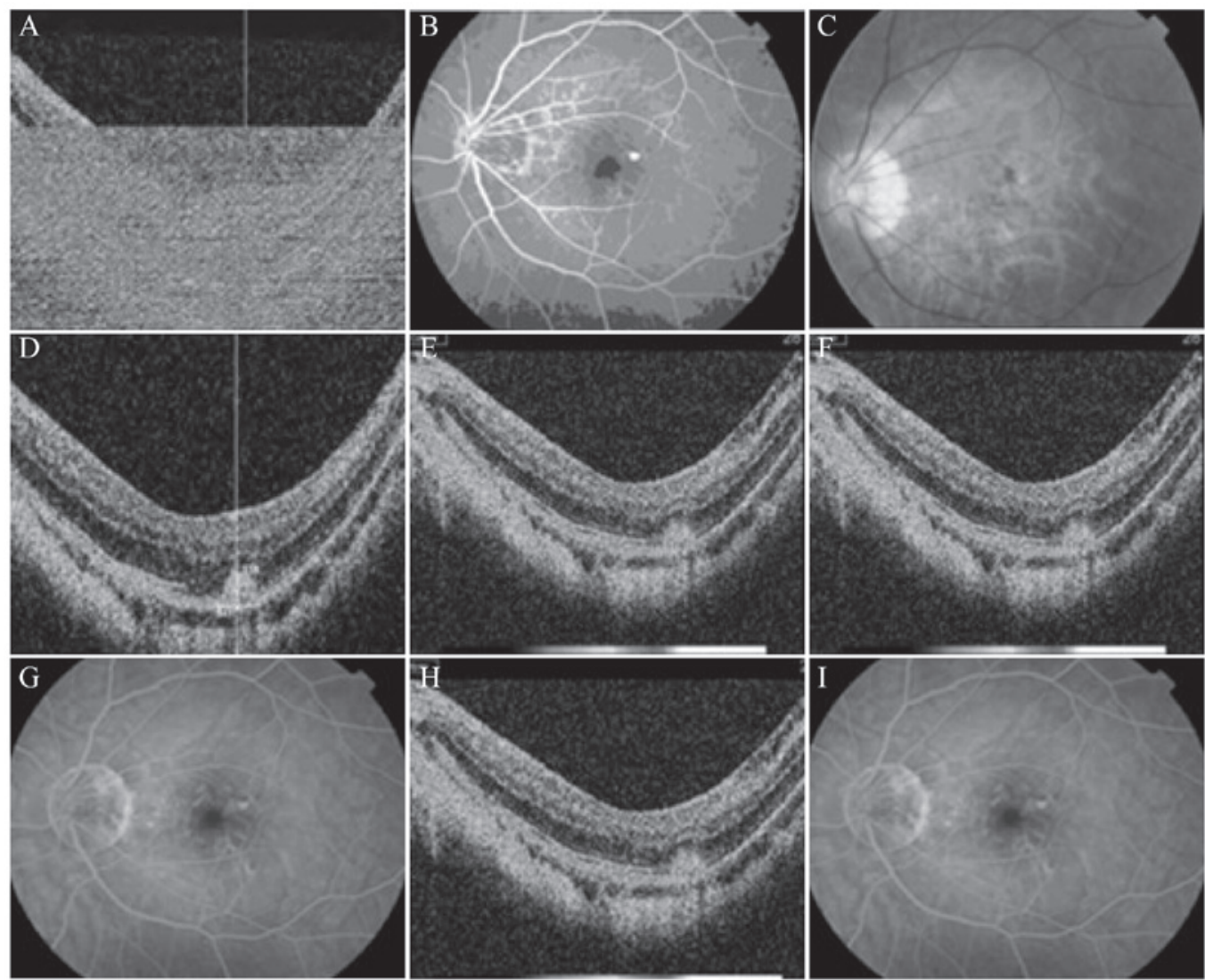

Figure 2. Case 1: FFA and OCT comparison chart prior to and subsequent to treatment. (A-C) The fundus image prior to treatment and the FFA showed parafovea high myopic CNV fluorescence leakage, and OCT showed CNV activity as low and medium signals in the lesions; the BCVA prior to treatment was 0.4. (D and E) FFA and OCT 1 month after the intravitreal injection of ranibizumab treatment; the BCVA was 0.5. (F and G) FFA and OCT 3 months after the intravitreal injection of ranibizumab treatment; the BCVA was 0.6. (H and I) FFA 6 months after the intravitreal ranibizumab injection(s) showed that the macular neovascular leakage had almost disappeared, and the high fluorescence leakage had turned into a weak fluorescence. OCT showed that the macular CNV was fibrotic and the reflection band turned from low and medium signals to high signals; the best-corrected vision was 0.6. FFA, fundus fluorescein angiography; OCT, optical coherence technology; CNV, choroidal neovascularization; BCVA, best-corrected visual acuity.
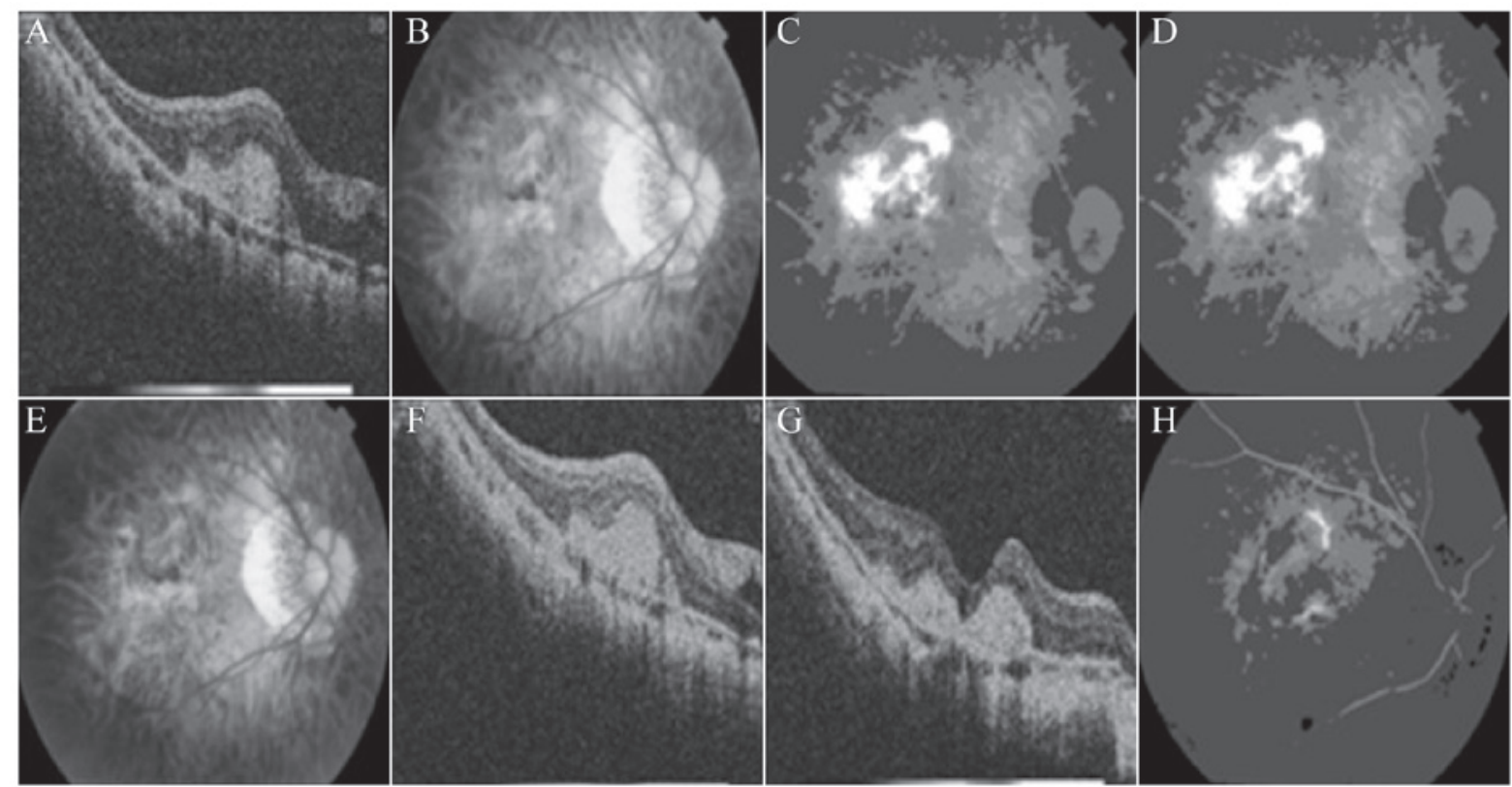

Figure 3. Case 2: FFA and OCT comparison chart prior to and subsequent to treatment. (A-D) The fundus image prior to treatment and FFA showed parafovea high myopic CNV fluorescence leakage, and OCT showed CNV activity as low and medium signals in the lesions; the best-corrected visual acuity prior to treatment was 0.02. (E-H) FFA and OCT 6 months after the intravitreal ranibizumab injection (best-corrected vision, 0.8 ). The subsequent follow-up revealed that the best-corrected vision had almost no change, and FFA revealed that the neovascular fluorescence-disc leakage turned into a weak-spot fluorescence. OCT indicated that the low and medium reflection signals of the CNV lesions had turned into high signals. FFA, fundus fluorescein angiography; OCT, optical coherence technology; $\mathrm{CNV}$, choroidal neovascularization. 
the full-length antibody (16). It can thus bind to VEGF-A with higher affinity, consequently blocking the cascade reaction caused by the combination of VEGF-A and VEGF receptors $\mathrm{R} 1 / \mathrm{R} 2$ on the vascular endothelium, and achieving the most beneficial biological treatment effect (17). Histopathologically, ranibizumab inhibits the proliferation of endothelial cells and vascular permeability and reduces inflammation and leakage, thereby preventing CNV formation and macular edema (18).

In the present study, the intravitreal injection of ranibizumab improved vision and reduced macular retinal thickness and fluorescein leakage in 60 out of 61 eyes. A slight visual improvement occurred in one eye only following four intravitreal injections of ranibizumab, and the best correction in vision was from 0.02 to 0.04 . The reason for this small improvement is unclear and further research is required to determine why certain eyes may not respond to this injection. Previous studies have assessed the number of treatments required and the post-treatment visual outcomes following intravitreal ranibizumab injection $(8,19)$. Figurska and Stankiewicz (19) reported an Early Treatment Diabetic Retinopathy Study (ETDRS) visual acuity improvement of two lines in a 55-year-old patient who received two intravitreal injections of ranibizumab; furthermore, the ETDRS visual acuity was improved by three lines in another 25-year-old patient who received three intravitreal ranibizumab injections. A similar study (8) reported that the average number of intravitreal ranibizumab injections required to treat pathological myopia-induced macular CNV was 2.2, and the average BCVAs were 0.01 (prior to the treatment) and 0.30 (subsequent to the treatment). In the present study, the average number of injections was 1.97 , and the average BCVAs were $0.02 \pm 0.01$ and $0.30 \pm 0.03$ prior to and subsequent to treatment, respectively. Compared with the aforementioned previous studies, fewer injections were administered and better vision improvements were obtained following the treatment. Different research programs and ethnic differences, as well as the differences among the patients and other conditions, may explain these variations in results between the present and prior studies. Although the suitability of the age-grouping method has been questioned (20), we postulated that age grouping would reflect the effect of age on the treatment effect. Regarding the injection number option, we adopted the pro re nata method, similar to Wakabayashi et al (13), in which the administration of further injections was determined based on the first injection curve effect. Few researchers have selected the classic treatment, which is suitable for exudative AMD; in the classic treatment, the decision of whether to re-treat is based upon the evaluation following three consecutive injections (21-23). Different treatment options in the previous studies may have been based on the facts that the leakage, edema and lesion areas of pathological myopia-induced CNV lesions are lower than those of exudative AMD, and the lesions are mainly located under the macula lutea. Regarding the association between pathological myopia-induced macular CNV lesion locations and the fovea centralis, the CNV lesions of 44 eyes were located under the macular fovea centralis, accounting for $72.1 \%$ (44/61) of cases in the present study.

The lacquer crack-like lesion represents the unique damage of pathological myopia-induced eyeground disease, and it is the marker of macular degeneration. Comparative observa- tions of indocyanine-green angiography (ICGA) and FFA have shown that the two techniques have a discovery rate of lacquer crack-like lesions of 89 and 38\%, respectively, indicating that ICGA can detect these lesions more effectively than can FFA (8). In the present study, lacquer cracks were detected in 40 out of 61 eyes $(65.6 \%)$ with pathological myopia-induced macular CNV. Among the 40 eyes, lacquer cracks were detected in 15 out of the contralateral eyes (37.5\%). This result has prompted us to pay increased attention to lacquer cracks in the treatment and research of pathological myopia. Patients in whom lacquer cracks are found in one eye should be considered to be at a high risk of lacquer cracks in the contralateral eye, and early intervention may be necessary. This subject is worthy of further investigation.

Regarding adverse reactions, 10 eyes exhibited subconjunctival thick hemorrhage with the bleeding being self-absorbed $\sim 1$ week later. An infectious weak anterior chamber reaction without the occurrence of endophthalmitis was observed in one eye, and the anterior chamber reaction was resolved following treatment with TobraDex and levofloxacin eye drops four times per day for 7 consecutive days. Increased intraocular pressure occurred in three cases, with an average increase of $5 \mathrm{mmHg}$. The eye pressures dropped to normal levels following treatment with timolol eye drops twice per day for 3 consecutive days, and the subsequent follow-up revealed no further increases in intraocular pressure. No cases of complicated cataract and retinal tear or detachment were observed, which may be primarily associated with the skill and proficiency of the surgeons.

In conclusion, intravitreal ranibizumab treatment showed high efficacy for pathological myopia-induced macular CNV; this treatment required few injections and resulted in few side effects, while achieving improvements in visual acuity and organ structure. This treatment therefore appears to be useful for this condition. The present study had several limitations, however: First, the sample size of 61 eyes was small, and further studies with a larger sample size are required; secondly, the follow-up period of 6 months was short, and controlled studies with a follow-up period of 1 or 2 years will be necessary; thirdly, this study lacked a control group, and a comparative study including other treatment methods would provide results that are more robust. In the previous discussions, although no comparative research was suggested regarding which treatment was optimal, there were studies that outlined the limitations of other methods $(14,15)$. Currently, only ranibizumab has been approved in China for the treatment of wet AMD, while it has not been approved for the treatment of pathological myopia-induced CNV, it is used outside of the specified indications. Although a previous study indicated that ranibizumab should become the first-line treatment for pathological myopia-induced CNV (24), a full explanation of the treatment should be provided and approval should be obtained from the Medical Ethics Committee and from the patient when the treatment is applied. Furthermore, the side effects of ranibizumab in treating pathological myopia-induced $\mathrm{CNV}$ in Asian populations require further study and confirmation. Since the follow-up times in this study were inconsistent and the average follow-up time was short, both the sample size and the follow-up time should be extended in the future, and an in-depth study should be performed with the purpose of 
obtaining information regarding recurrence and injection times at different periods, among other observations. The present study and its recommendation, which may be novel for some, arose from questions that have emerged from developments in medical science. Thus, a novel method that is more effective, safe and reliable for the treatment of pathological myopia-induced CNV may be possible in the near future.

\section{References}

1. Silva R: Myopic maculopathy: A review. Ophthalmologica 228 : 197-213, 2012.

2. Sawada A, Tomidokoro A, Araie M, Iwase A and Yamamoto T; Tajimi Study Group: Refractive errors in an elderly Japanese population: The Tajimi study. Ophthalmology 115: 363-370, 2008.

3. Cheng CY, Hsu WM, Liu JH, Tsai SY and Chou P: Refractive errors in an elderly Chinese population in Taiwan: The Shihpai Eye Study. Invest Ophthalmol Vis Sci 44: 4630-4638, 2003.

4. Rosenfeld PJ, Brown DM, Heier JS, et al; MARINA Study Group: Ranibizumab for neovascular age-related macular degeneration. N Engl J Med 355: 1419-1431, 2006.

5. Pece A, Vadalà M, Isola V and Matranga D: Photodynamic therapy with verteporfin for juxtafoveal choroidal neovascularizzation in pathologic myopia: A long-term follow-up study. Am J Ophthalmol 143: 449-454, 2007.

6. Martin DF, Maguire MG, Ying GS, Grunwald JE, Fine SL and Jaffe GJ; CATT Research Group: Ranibizumab and bevacizumab for neovascular age-related macular degeneration. N Engl J Med 364: 1897-1908, 2011.

7. Gharbiya M, Allievi F, Mazzeo L and Gabrieli CB: Intravitreal bevacizumab treatment for choroidal neovascularization in pathologic myopia: 12-month results. Am J Ophthalmol 147: 84-93, e1, 2009.

8. Vadalà M, Pece A, Cipolla $\mathrm{S}$, et al: Is ranibizumab effective in stopping the loss of vision for choroidal neovascularisation in pathologic myopia? A long-term follow-up study Br J Ophthalmol 95: 657-661, 2011.

9. Otsuka K, Imai H, Shimoyama T, Nagai T, Honda S and Azumi A: Recurrence of macular hole retinal detachment after intravitreal ranibizumab injection for the treatment of choroidal neovascularization from the remaining macular hole edge. Case Rep Ophthalmol 3: 424-427, 2012.

10. Lorenzo D, Arias L, Alcubierre R, et al: Intravitreal ranibizumab for choroidal neovascularization secondary to pathological myopia: 12-month follow-up. Ophthalmologica 226: 103-109, 2011.
11. Yoshida T, Ohno-Matsui K, Yasuzumi K, et al: Myopic choroidal neovascularization: A 10-year follow-up. Ophthalmology 110: 1297-1305, 2003.

12. Hayashi K, Shimada N, Moriyama M, Hayashi W, Tokoro T and Ohno-Matsui K: Two-year outcomes of intravitreal bevacizumab for choroidal neovascularization in Japanese patients with pathologic myopia. Retina 32: 687-695, 2012.

13. Wakabayashi T, Ikuno Y and Gomi F: Different dosing of intravitreal bevacizumab for choroidal neovascularization because of pathologic myopia. Retina 31: 880-886, 2011.

14. Mateo C, Moreno J, Rosales G, et al: Two-year results of macular translocation with scleral infolding in myopic choroidal neovascularisation. Semin Ophthalmol 19: 29-42, 2004.

15. Ladas ID, Moschos MM, Rouvas AA, Karagiannis DA and Kokolakis SN: Lacquer crack formation after photodynamic therapy. Eur J Ophthalmol 13: 729-733, 2003.

16. Ferrara N, Damico L, Shams N, Lowman H and Kim R: Development of ranibizumab, an anti-vascular endothelial growth factor antigen binding fragment, as therapy for neovascular age-related macular degeneration. Retina 26: 859-870, 2006.

17. Chen Y, Wiesmann C, Fuh G, et al: Selection and analysis of an optimized anti-VEGF antibody: Crystal structure of an affinity-matured Fab in complex with antigen. J Mol Biol 293: 865-881, 1999.

18. Krzystolik MG, Afshari MA, Adamis AP, et al: Prevention of experimental choroidal neovascularization with intravitreal anti-vascular endothelial growth factor antibody fragment. Arch Ophthalmol 120: 338-346, 2002.

19. Figurska $M$ and Stankiewicz A: Anti-VEGF therapy in the treatment of myopic macular choroidal neovascularization - case report. Klin Oczna 110: 387-391, 2008 (In Polish).

20. Ng DS, Kwok AK and Chan CW: Anti-vascular endothelial growth factor for myopic choroidal neovascularization. Clin Experiment Ophthalmol 40: e98-e110, 2012.

21. Wu TT and Kung YH: The 12-month outcome of three consecutive monthly intravitreal injections of ranibizumab for myopic choroidal neovascularization. J Ocul Pharmacol Ther 28: 129-133, 2012.

22. Lai TY, Chan WM, Liu DT and Lam DS: Intravitreal ranibizumab for the primary treatment of choroidal neovascularization secondary to pathologic myopia. Retina 29: 750-756, 2009.

23. Calvo-Gonzalez C, Reche-Frutos J, Donate J, Fernandez-Perez C and Garcia-Feijoo J: Intravitreal ranibizumab for myopic choroidal neovascularization: Factors predictive of visual outcome and need for retreatment. Am J Ophthalmol 151: 529-534, 2011.

24. Neelam K, Cheung CM, Ohno-Matsui K, Lai TY and Wong TY: Choroidal neovascularization in pathological myopia. Prog Retin Eye Res 31: 495-525, 2012. 